ESAIM: PROCEEDINGS, July 2007, Vol.18, 133-142

Jean-Frédéric Gerbeau \& Stéphane Labbé, Editors

\title{
A GLUEY PARTICLE MODEL
}

\author{
B. MAURY ${ }^{1}$
}

\begin{abstract}
We present here a new framework to handle the short-range interaction between rigid bodies in a viscous, incompressible fluid. This framework is built as the vanishing viscosity limit of a lubrication model. We restrict ourselves here to the case of a single particle and a rigid wall. Our approach is based on a standard first-order approximation for the lubrication force between two rigid bodies, where a small parameter $\varepsilon$ plays the role of the underlying fluid viscosity. We establish convergence when $\varepsilon$ goes to 0 of a subsequence of trajectories towards a solution to a problem of the hybrid type: it relies on two distincts states, unglued and glued, the latter being described by a new variable $\gamma$ which expresses in a way the asymptotic smallness of the distance, and which plays the role of an adhesion potential. The limit problem has a surprising property: although it is well-posed in many situations, uniqueness does not generally hold as soon as left-hand clusters of contact times are allowed. Some prospective extensions of this model (other types of singularities, roughness of surfaces, macroscopic version) are proposed.
\end{abstract}

Résumé. Nous présentons ici un nouveau cadre pour gérer l'interaction à courte portée entre corps rigides baignant dans un fluide visqueux. Notre modèle est construit comme la limite à viscosité évanescente d'un modèle de lubrification. Nous nous restreignons ici au cas d'une sphère unique au voisinage d'une paroi plane. Le point de départ de notre approche est ainsi un modèle qui estime au premier ordre les forces de lubrification entre une sphère et un plan en quasi-contact, modèle dans lequel un petit paramètre $\varepsilon$ joue le rôle de la viscosité du fluide environnant. Nous montrons la convergence, quand $\varepsilon$ tend vers 0 , d'une sous-suite de solutions vers une solution d'un problème de type hybride. Ce modèle limite se base sur la définition de deux états, libre et collé, ce dernier état étant décrit par une nouvelle variable $\gamma$ qui représente d'une certaine manière la petitesse asymptotique de la distance, et qui joue le rôle d'un potentiel d'adhésion. Le problème limite jouit d'une propriété surprenante : bien qu'il soit bien posé dans un grand nombre de situations, on n'a pas unicité en général dès que l'on sort du cadre d'instants de contact isolés. Nous présentons pour finir des extensions possibles de ce travail (autres types de singularité, prise en compte de la rugosité, version macroscopique du modèle).

\section{Introduction, Finite Viscosity LUBRICATION MODEL}

We are interested in the behaviour, as $\varepsilon$ goes to 0 , of the solution $q_{\varepsilon}$ to

$$
\mid \begin{aligned}
& \ddot{q}_{\varepsilon}=-\varepsilon \frac{\dot{q}_{\varepsilon}}{q_{\varepsilon}}+f(t), \\
& q_{\varepsilon}(0)=q^{0}>0, \quad \dot{q}_{\varepsilon}(0)=u^{0},
\end{aligned}
$$

where $f$ is a given forcing term. It models the motion of a solid sphere of unit mass in a viscous fluid, subject to move horizontally on the $x$-half line, in the neighbourhood of the plane $x=0$ corresponding to a solid wall (see

${ }^{1}$ Laboratoire de Mathématiques, Université Paris Sud, 91405 Orsay cedex. e-mail: Bertrand.Maury@math.u-psud.fr

(c) EDP Sciences, SMAI 2007 


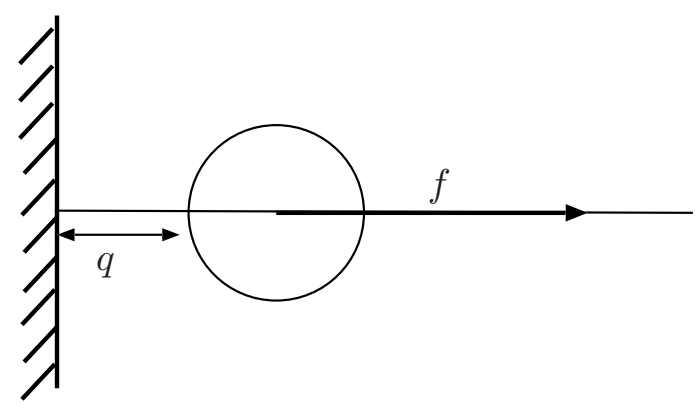

Figure 1. Geometry.

Fig. 1). The interaction force which results from the three dimensional lubrication flow in the gap between a ball and a plane can indeed be proved to behave like the product of the reciprocal of the wall-particle distance, the relative velocity, and the viscosity of the surrounding fluid (see $[5,7])$.

The main purpose of this paper is to establish convergence of the solution to this ODE, when $\varepsilon$ goes to 0 , towards a solution to an evolution problem of the hybrid type. Indeed, the description of the asymptotic behaviour calls for the use of an extra variable $\gamma$ which is activated when contact occurs. This new variable $\gamma$ makes it possible to include memory effects in the limit model: it keeps track of the sollicitations undergone by the sphere as long as it is stuck to the wall. As we shall see, $\gamma$ measures in a way the asymptotic smallness of the wall-particle distance, and yet it follows the same equation as the one the velocity would obey if there were no obstacle.

As a first step, we prove well-posedness of Problem (1), which is a straightforward consequence of the fact that the sphere never touches the wall:

Proposition 1.1. Let $\varepsilon>0$ and $f \in L_{l o c}^{1}\left(\mathrm{R}^{+}\right)$be given. Then Problem (1) admits a unique global solution $q_{\varepsilon} \in W_{l o c}^{1, \infty}\left(\mathrm{R}^{+}\right)$.

Proof. The local Lipschitz character of $(q, u) \mapsto u / q$ in $] 0,+\infty[\times \mathrm{R}$ ensures existence and uniqueness of a maximal solution to (1), defined over the time interval [0, $\tau$ [. Lipschitz regularity of this solution is a consequence of the standard energy estimate combined with Gronwall Lemma:

$$
\frac{1}{2}\left|\dot{q}_{\varepsilon}\right|^{2} \leq \frac{1}{2}\left|\dot{q}_{\varepsilon}\right|^{2}+\varepsilon \int_{0}^{t} \frac{\left|\dot{q}_{\varepsilon}\right|^{2}}{q_{\varepsilon}} \leq \frac{1}{2}\left|u^{0}\right|^{2}+\int_{0}^{t}|f|\left|\dot{q}_{\varepsilon}\right| \Longrightarrow\left|\dot{q}_{\varepsilon}\right| \leq\left|u^{0}\right|+\int_{0}^{t}|f|
$$

From this estimate, we also get that the velocity $\dot{q}_{\varepsilon}$ cannot blow up within a finite time, so that, if $\tau<\infty$, then necessarily $q_{\varepsilon}$ goes to 0 as $t$ goes to $\tau^{-}$. Integration of (1) gives

$$
\dot{q}_{\varepsilon}(t)=u^{0}-\varepsilon \ln \left(\frac{q_{\varepsilon}(t)}{q^{0}}\right)+\int_{0}^{t} f(s) d s
$$

so that $q_{\varepsilon} \rightarrow 0$ implies $\dot{q}_{\varepsilon}(t) \mapsto+\infty$, hence a contradiction.

Remark 1.2. Note that this no-contact property does no longer hold as soon as the lubrication force is weakened. For instance, if $-\varepsilon \dot{q}_{\varepsilon} / q_{\varepsilon}$ is replaced by $-\varepsilon \dot{q}_{\varepsilon} / q_{\varepsilon}^{1-\eta}$, with $\eta>0$, then contact may occur within a finite time, and the previous proposition, as well as the rest of the paper, is invalidated. On the other hand, more singular forces like $-\varepsilon \dot{q}_{\varepsilon} / q_{\varepsilon}^{1+\eta}$ can be taken into account (see Section 5.1). 


\section{Asymptotic Behaviour}

Let us denote by $I$ the time interval ]0,T[. Our aim is to establish convergence (up to a subsequence) of $q_{\varepsilon}$ towards a function $q$ which is a solution to a problem of the hybrid type on $I$. We shall first describe the limit problem in three different forms, which are formally equivalent. The first one, on which the convergence theorem 2.1 shall be based upon, is the following:

$$
\mid \begin{aligned}
& \text { Find } q \in W^{1, \infty}(I), \quad \gamma \in L^{\infty}(I), \text { such that } \\
& \dot{q}+\gamma=\tilde{u}=u^{0}+\int_{0}^{t} f(s) d s \quad \text { a.e. in } I, \\
& \gamma \leq 0, q \geq 0, \quad q \gamma=0 \quad \text { a.e. in } I, \\
& q(0)=q^{0}>0, \quad \dot{q}(0)=u^{0} .
\end{aligned}
$$

As we shall see in Section 4, this problem may admit multiple solutions in general, and it is not a matter of regularity conditions on $f$ : as suggested by counter-examples for granular flow models, only analyticity can be expected to provide uniqueness, $f \in C^{\infty}$ being not sufficient (see $[1,10,11]$ ).

Yet, it is somehow well-posed in the sense that, in many situations, a solution can be built in a unique way. Let us describe a heuristic procedure to build a solution. At first, the system behaves as if there were no wall, $q$ is positive and therefore $\gamma$ is 0 . Note that $q$ cannot drop abruptly to 0 , for continuity is required. Let us assume that the unconstrained pathline would become negative for the first time right after some time $\tau_{1}>0$. It is not possible for $q$ to become negative, and therefore $q$ is stuck to the value 0 , whereas $\gamma$ is activated right after $\tau_{1}$, and is defined by $\gamma=\tilde{u}$. As far as $\gamma$ remains negative, $q$ remains glued on the wall, and $\gamma$ can not jump abruptly from a negative value to 0 because, if it did, $q$ would restart with a negative velocity $\tilde{u}$, hence $q$ would actually enter the wall. Therefore, $\gamma$ goes smoothly to 0 from below, and when it hits 0 at time $\tau_{2}, q$ takes off from the wall. Fig. 2 presents an example of such a behaviour. Note that this approach cannot be carried out in the neighbourhood of cluster points of hitting times.

Problem (3) can be expressed in the spirit of granular flow models (see $[1,10]$ ). The formulation we propose now is close to (3), with the difference that the interaction force between the wall and the particle is explicited. based on this second formulation. It reads

$$
\mid \begin{aligned}
& \text { Find } q \in W^{1, \infty}(I) \text { with } \dot{q} \in \mathrm{BV}(I), \quad \gamma \in \mathrm{BV}(I), \quad \mu \in \mathcal{M}(I), \quad \text { such that } \\
& \ddot{q}=f+\mu \text { in } \mathcal{M}(I) \\
& \operatorname{supp}(\mu) \in\{t, q(t)=0\} \\
& \dot{q}^{+}=P_{C_{q}} \dot{q}^{-} \\
& \dot{\gamma}=-\mu \\
& \gamma \leq 0, q \geq 0, q \gamma=0 \quad \text { a.e. in } I, \\
& q(0)=q^{0}>0, \dot{q}(0)=u^{0},
\end{aligned}
$$

where $\mathcal{M}(I)$ denotes the set of bounded measures on $I, \mathrm{BV}(I)$ the set of functions with bounded variation over $I, \dot{q}^{+}$(resp. $\dot{q}^{-}$), is the right limit (resp. left limit) of $\dot{q}$, and $C_{q}$ is the convex cone of feasible velocities, defined by

$$
C_{q}=\mid \begin{array}{lll}
\mathrm{R} & \text { if } & q>0, \\
\mathrm{R}^{+} & \text {if } & q=0 \text { and } \gamma^{-}=0, \\
\{0\} & \text { if } & q=0 \text { and } \gamma^{-}<0 .
\end{array}
$$

The theoretical framework on which similar formulations (for granular flows with non-gluey particles) are based is thoroughly described in $[1,10]$. Let us simply note here that right and left limits of $\dot{q}$ and $\gamma$ are well-defined as both functions have bounded variation. 
Before stating the convergence result, let us mention a third, event-driven formulation, which formalizes the heuristic procedure to build up a solution given above. It is based on the introduction of two distinct states for the system, indexed by 0 (unglued, the particle does not touch the wall, $q>0$ ) and 1 (glued, the particle is in contact with the wall, $q=0$ and $\gamma<0)$. Borrowing the terminology of [6], we introduce the set of states $Q$, the collection of domains $(D)$, of forcing terms $(F)$, of jump sets $(G)$, and the transition maps $R_{01}$ and $R_{10}$ :

$$
\begin{aligned}
& Q=\{0,1\}, \quad D=\left(D_{0}, D_{1}\right)=\left(\left(\mathrm{R}^{+} \times \mathrm{R}\right), \mathrm{R}^{-}\right), X_{0}=\left[\begin{array}{c}
q \\
u
\end{array}\right], X_{1}=\gamma, \\
& F=\left(F_{0}, F_{1}\right)=\left(\left[\begin{array}{c}
u \\
f
\end{array}\right],[f]\right), \\
& G_{01}=\left[\begin{array}{l}
0 \\
\mathrm{R}^{-}
\end{array}\right], G_{10}=\{0\}, \quad R_{01}\left(\left[\begin{array}{l}
0 \\
u
\end{array}\right]\right)=u, R_{10}(0)=\left[\begin{array}{l}
0 \\
0
\end{array}\right] .
\end{aligned}
$$

This hybrid framework describes the following behaviour: Starting from $q^{0}>0$ at $t=0$, the system is in state 0 , so that the domain is $D_{0}$, and the state variable $X_{0}=[q, u]^{t}$ verifies

$$
\dot{X}_{0}=F_{0}\left(X_{0}\right), \quad \text { namely }\left[\begin{array}{c}
\dot{q} \\
\dot{u}
\end{array}\right]=\left[\begin{array}{c}
u \\
f
\end{array}\right]
$$

As soon as $G_{01}$ is hit (i.e. as soon as $q=0$ ), at a certain time $\tau_{1}>0$, the system switches to states 1 , and the new variable $X_{1}$, which is $\gamma$ in the previous notations, is set to $R_{01}\left(X_{0}\left(\tau_{1}^{-}\right)\right)$, which is the value of $u$ right before the contact. The system continues with the new dynamic

$$
\dot{X}_{1}=F_{1}\left(X_{1}\right), \quad \text { namely } \dot{\gamma}=f
$$

until $G_{10}=\{0\}$ is hit, making the system switch back to state 0 (the particle is free to take off the wall).

We may now state the convergence result, which refers to the formulation (3) of the limit problem.

Theorem 2.1. Let $q^{0}>0, u^{0} \in \mathrm{R}$, a time interval $\left.I=\right] 0, T\left[\right.$ and $f \in L^{1}(I)$ be given. We denote by $q_{\varepsilon} \in W^{1, \infty}(I)$ the unique solution to (1) in $\bar{I}$, and we set $\gamma_{\varepsilon}=\varepsilon \ln q_{\varepsilon}$. When $\varepsilon$ goes to 0 , there exists a subsequence, still denoted by $\left(q_{\varepsilon}\right), q \in W^{1, \infty}(I), \gamma \in L^{\infty}(I)$, such that

$$
\begin{array}{llll}
q_{\varepsilon} & \longrightarrow & q & \text { uniformly }, \\
\gamma_{\varepsilon} & \star & \gamma & \text { in } L^{\infty} \text { weak }-\star
\end{array}
$$

and the couple $(q, \gamma)$ is a solution to Problem (3).

Proof. Boundedness of $\left(q_{\varepsilon}\right)$ in $W^{1, \infty}(I)$ is a direct consequence of (2) (see the proof of Prop. 1.1). Consequently, one can extract a subsequence (still denoted by $q_{\varepsilon}$ ) such that $q_{\varepsilon}$ converges uniformly to some $q \in W^{1, \infty}$, and $\dot{q}_{\varepsilon}$ converges to $u=\dot{q}$ in the weak- $\star$ sense.

Let $\gamma_{\varepsilon}$ be defined as $\varepsilon \ln q_{\varepsilon}$. Straightforward computations give

$$
\dot{q}_{\varepsilon}+\gamma_{\varepsilon}=u^{0}+\gamma_{\varepsilon}^{0}+\int_{0}^{t} f
$$

where $\gamma_{\varepsilon}^{0}=\gamma_{\varepsilon}(0)=\varepsilon \ln q^{0}$ goes to 0 with $\varepsilon$. As a consequence, $\gamma_{\varepsilon}$ converges weak- $\star$ in $L^{\infty}$ towards $\gamma \in L^{\infty}$ such that

$$
\dot{q}+\gamma=u^{0}+\int_{0}^{t} f=\tilde{u}(t)
$$


The next step consists in proving that $q$ is such that $\dot{q}=\tilde{u}$ in $I_{0}=\{t, q(t)>0\}$. To that purpose we introduce, for any $\eta>0$, the set $I_{\eta}(q)=\{t \in] 0, T[, q(t)>\eta\}$. As $q_{\varepsilon}$ converges uniformly to $q$, one has $I_{\eta}(q) \subset I_{\eta / 2}\left(q_{\varepsilon}\right)$ for $\varepsilon$ sufficiently small. As a consequence, $\gamma_{\varepsilon}=\varepsilon \ln q_{\varepsilon}$ goes uniformly to 0 on $I_{\eta}(q)$, thus $\dot{q}_{\varepsilon}$ converges uniformly towards the unconstrained velocity $\tilde{u}$ in $I_{\eta}(q)$, for all $\eta>0$. The limit $q$ is therefore $C^{1}$ on $I_{0}$, with $\dot{q}=\tilde{u}$, and $\gamma$ is identically 0 on $I_{0}$.

On $I_{0}^{c}(q)=\{t, q(t)=0\}, q$ is constant, so that $\dot{q}=0$ almost everywhere, thus $\gamma=\tilde{u}$ a.e. Beside, as $\gamma_{\varepsilon}$ is negative as soon as $q_{\varepsilon}$ is less than 1 , one has $\gamma \leq 0$ on $I_{0}^{c}$.

\section{Numerical illustration}

This section presents some computations to illustrate the convergence of $q_{\varepsilon}$ (resp. $\gamma_{\varepsilon}$ ) towards $q$ (resp. $\gamma$ ). As it is not the point of this paper to go deep into numerical considerations, we shall simply describe the algorithms we used to run the numerical tests. Firstly, the numerical computation of (1), for $\varepsilon>0$, is based on a semi-implicit Euler scheme applied to the integrated equation for $\gamma_{\varepsilon}=\varepsilon \ln q_{\varepsilon}$ (we drop the subscript $\varepsilon$ for readability reasons):

$$
\dot{q}=-\varepsilon \ln \frac{q}{q^{0}}+\tilde{u} \Longrightarrow \dot{\gamma}=\varepsilon e^{-\gamma / \varepsilon}(\tilde{u}-\gamma)
$$

and the latter equation is discretized by

$$
\frac{\gamma^{n+1}-\gamma^{n}}{h}=\varepsilon e^{-\gamma^{n} / \varepsilon}\left(\tilde{u}\left(t^{n+1}\right)-\gamma^{n+1}\right)
$$

which can also be written

$$
\gamma^{n+1}=\theta \gamma^{n}+(1-\theta) \tilde{u}\left(t^{n+1}\right) \quad \text { with } \quad \theta=\frac{1}{1+h \varepsilon e^{-\gamma^{n} / \varepsilon}} .
$$

Note how the latter form exhibits the hybrid behaviour: when $\theta$ is close to 1 ( $\gamma$ close to 0 , unglued case), $\gamma^{n}$ does not vary much, whereas when $\theta$ is close to 0 ( $\gamma$ negative, glued case), the approximation of $\gamma$ follows $\tilde{u}$.

As for the hybrid formulation, we used the following scheme: with $t^{n}=n h$. Assuming $q^{n}$, $u^{n}$, and $\gamma^{n}$ are known at time step $t^{n}$, the next values are computed according to the following alternative (depending on whether the contact is active or not):

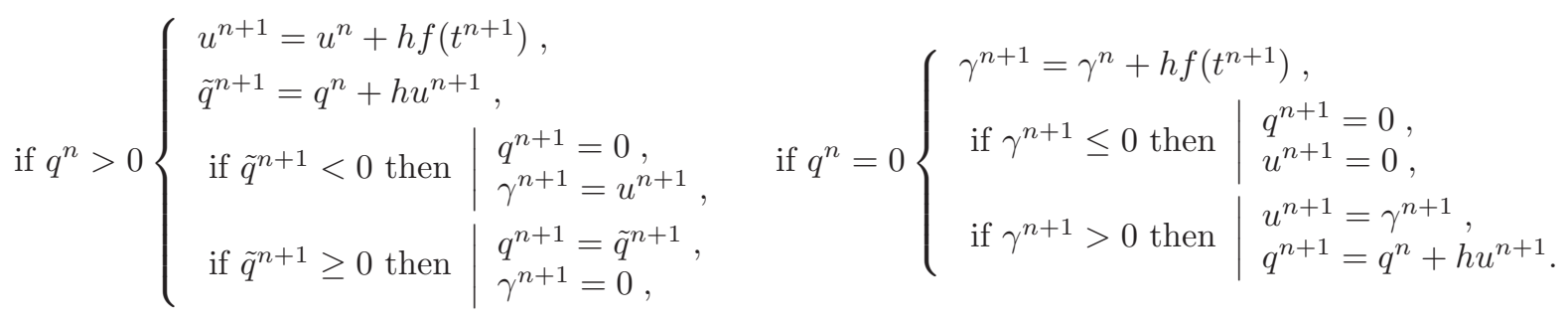

Figure 2 corresponds to the right-hand side

$$
f=-1_{] 0,2[}+1_{] 2,5[}
$$

We plotted the functions $q$ and $\gamma$, solutions to the limit problem (3), and $q_{\varepsilon}, \gamma_{\varepsilon}$, for $\varepsilon=0.25$.

Remark 3.1. Note that very small values of $q_{\varepsilon}$ can be reached, which may harm the computations. As an example, for $\varepsilon=10^{-2}$, the minimal value for $q$ is about $10^{-87}$. For $\varepsilon=2.10^{-3}$, the distance $q$ drops below the smallest real number which can be stored by standard numerical softwares $\left(10^{-324}\right.$ with Scilab). Concerning this non-physical value for the gap (computed wall-particle distances are likely to drop far below intermolecular distances, which rules out the lubrication model on which our approach is based), see Section 5.2. 


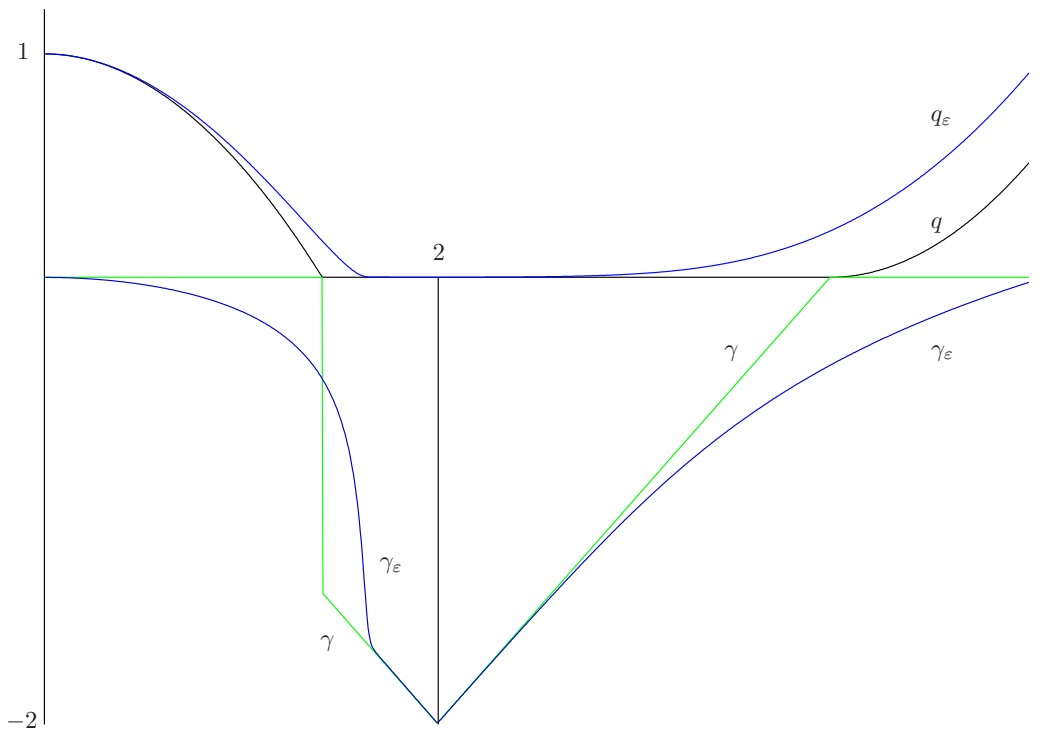

Figure 2. Convergence of $q_{\varepsilon}$ towards $q$.

\section{NON-UNIQUENESS FOR THE LIMIT PROBLEM}

The event-driven formulation (5) suggests well-posedness in terms of uniqueness. We show here that uniqueness no longer holds as soon as collisions cannot be handled as separate events. The example we propose exhibits indeed a cluster point (the origin) of collisions. It presents some similarities with the counter-example to uniqueness for elastic impact problems which was proposed in [11] (see also [1] for a precise description of similar constructions). In this section we shall say that $q$ is a solution to (3) if there exists $\gamma$ such that $(q, \gamma)$ is a solution to (3).

The construction is based on the following function $\rho$

$$
x \in] 1,2\left[\longmapsto \rho(x)=1_{] 1,1+\xi[}-\alpha 1_{] 1+\xi, 2[}\right.
$$

where $\alpha>0$ and $\xi \in] 0,1[$ are parameters, the value of which shall be set later. This function is to be used, together with translated copies of itself at different time scales, to build an oscillatory forcing term with infinite frequency at $0^{+}$. The generating function $\rho$ corresponds to a pull-up force during time $\xi$, succeded by a pushdown force during $1-\xi$. Consider now the forcing term $F$ defined in the interval $] 1,4[$ as

$$
F(x)=\rho(x) 1_{] 1,2[}+\rho\left(\frac{x}{2}\right) 1_{] 2,4[} .
$$

Note that $F$ is such that $F(2 x)=F(x)$ for $x \in] 1,2[$ (this property will be essential in the following). Now let us admit for the moment that the formerly introduced parameters $\alpha$ and $\xi$, and a new parameter $\tau$, can be chosen in such a way that the solution $Q$ to (3) on the time interval $] 1,4[$ associated to the right-hand side $F$, 
and which is such that $Q \equiv 0$ on $] 1,1+\tau]$ and $Q>0$ in the right neighbourhood of $1+\tau$, is such that (see Fig. 3 for an example of function $Q$ corresponding to such a choice):

$$
\begin{aligned}
& \text { The right end of its support is } 4-\eta \text { with } \eta>0 \\
& \text { If we denote by }-\beta<0 \text { the left-limit of } \dot{Q} \text { at } 4-\eta \text {, one has } \beta+\alpha \eta=4 \tau \\
& \text { It does not hold } Q(2 x)=Q(x) \text { in }] 1,2[
\end{aligned}
$$

The last property shall be essential to distinguish the two solutions which we plan to build.

We now define the right-hand side $f$ as

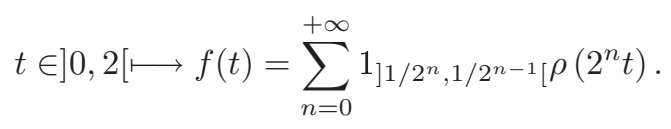

Note that $f(2 t)=f(t)$ for $t \in] 0,1\left[\right.$, and that $f$ is bounded and measurable, so that $f \in L^{1}(0,2)$.

Putting off the proof of the existence of appropriate parameters $\alpha, \xi$, and $\tau$ until the end of this section (see Lemma 4.2), we may now state the non-uniqueness result. Recall that $q$ is said to be a solution to (3) if there exists $\gamma$ such that $(q, \gamma)$ is a solution to (3). Note that $\gamma$ can be computed straightforwardly as soon as $q$ is given.

Proposition 4.1. Let $f$ be defined by (9), and let $Q \in C^{0}(] 1,4[)$ be built as indicated above. Then both functions

$$
t \in] 0,4\left[\longmapsto q_{1}(t)=\sum_{n=0}^{+\infty} \frac{1}{2^{4 n}} 1_{11 / 2^{2 n}, 1 / 2^{2 n-2}[} Q\left(2^{2 n} t\right)\right.
$$

and

$$
t \in] 0,2\left[\longmapsto q_{2}(t)=\sum_{n=0}^{+\infty} \frac{1}{2^{2(2 n+1)}} 1_{1 / 2^{2 n+1}, 1 / 2^{2 n-1}[} Q\left(2^{2 n+1} t\right)\right.
$$

are distinct solutions to the limit problem (3), with initial conditions $q(0)=0, \dot{q}(0)=0, \gamma(0)=0$.

Proof. Let us prove that $q_{1}$ is a solution to (3) in ]0,4[ (the proof for $q_{2}$ is similar). It amounts to show that $q_{1}$ is a solution on every subinterval $] 1 / 2^{2 n}, 1 / 2^{2 n-2}$ [ with adapted initial conditions, and that those conditions are provided by the behaviour in the left adjacent subinterval $] 1 / 2^{2 n+2}, 1 / 2^{2 n}[$. Now consider the intervals $] 1 / 4,1[$ and $] 1,4[$. Firstly, at times $t$ in $] 1 / 4,1\left[\right.$ where $q_{1}$ is not 0 , one has $q_{1}(t)=Q(4 t) / 16$, so that

$$
\ddot{q}_{1}(t)=\ddot{Q}(4 t)=F(4 t)=f(2 t)=f(t) .
$$

It remains to show that solutions on $] 1 / 4,1[$ and $] 1,4[$ can be concatenated to form a solution overall $] 1 / 4,4[$. At time $1-\eta / 4, q_{1}$ hits 0 with a velocity $-\beta / 4$, which activates $\gamma$. The adhesion potential $\gamma$ decreases then linearly with a slope $-\alpha$, reaching $-\beta / 4-\alpha \eta / 4$ at time 1 . It increases then with slope 1 , so that $\gamma$ hits 0 at time $1+\tau$, thanks to the second assumption of (8), which ensures connection with the solution $Q$ in the time interval $] 1,4[$. A similar proof can be made with all couples of consecutive time subintervals.

It remains to establish the existence of appropriate parameters.

Lemma 4.2 (Existence of $Q$ ). There exist parameters $\tau>0, \xi>0$, and $\alpha>0$ such that the solution $Q$ to (3) on $] 1,4[$, with a right-hand side defined by $F$ (see (7)), and which is such that $Q \equiv 0$ on $] 1,1+\tau]$ and $Q>0$ in the right neighbourhood of $1+\tau$, verifies the set of assumptions (8).

Proof. We fix $\alpha=1.8, \xi=0.54$, and we denote by $Q_{i}$ the solution associated to the triplet of parameters $\left(\tau_{i}, \alpha, \xi\right)$, with $\tau_{1}=0.29$ and $\tau_{2}=0.30$. With obvious notations, it can be established with the help of numerical computations that $\beta_{1}+\alpha \eta_{1}-4 \tau_{1}$ is positive, whereas the same quantity for the second set of parameters is 


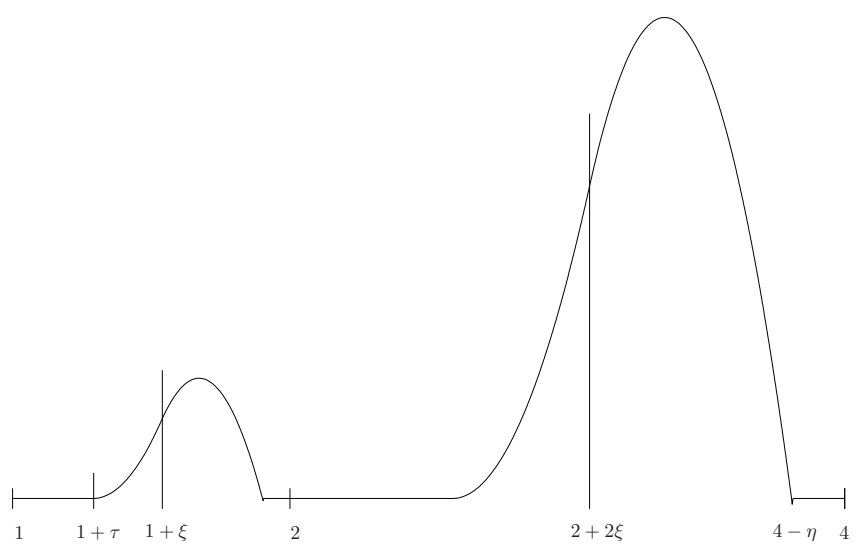

Figure 3. Function $Q$.

negative. By continuity, there exists a value $\tau \in] 0.29,0.30[$ such that the identity $\beta+\alpha \eta-4 \tau=0$ holds true for the solution associated to the triplet of parameters $(\tau, \alpha, \xi)$. The shape of this solution is represented in Fig. 3.

Remark 4.3. The two solutions we built are obviously indistinguishable: as $q_{1}(2 t)=4 q_{2}(t)$, one cannot expect any entropy-like criterium to be able to select between both.

\section{Extensions of THE MODEL}

We end this paper with some comments on the possible extensions of this model. The first point we address here is a straightforward generalization of the convergence result. The other points are more prospective, and have not undergone a proper mathematical treatment.

\subsection{Alternative lubrication models}

The approach we propose can be extended straighforwardly to the more general setting :

$$
\ddot{q}_{\varepsilon}=-\varepsilon \frac{\dot{q}_{\varepsilon}}{\varphi\left(q_{\varepsilon}\right)}+f(t)
$$

where $q \longmapsto \varphi(q)>0$ is such that $\phi(q)=\int_{q}^{1} \frac{1}{\varphi} \rightarrow+\infty$ as $q \longrightarrow 0$.

In case the latter integral is convergent, contacts may occur, which makes our approach inappropriate. In case the integral is divergent, defining $\gamma_{\varepsilon}=\varepsilon \phi\left(q_{\varepsilon}\right)$, we get as in Theorem 2.1 convergence of $\left(q_{\varepsilon}, \gamma_{\varepsilon}\right)$ towards $(q, \gamma)$, solution to (3). This extension covers the two-dimensionnal lubrication model (particles are in fact parallel cylinders), for which $\varphi(q)=q^{-3 / 2}($ see $[7])$.

Remark 5.1. Note that the asymptotic behaviour does not depend on the model, as soon as the integral of $1 / \varphi$ is divergent. 


\subsection{Collisions}

All this approach is based on the lubrication model (1), according to which the rigid body never touches the wall (see proposition 1.1). It is common knowledge, though, that in many situations, contacts actually occur. Some factors may explain this discrepancy, and among them:

(1) bodies and walls are not smooth (smoothness is an essential assumption to establish the model (1)), they present a certain roughness which rules out the lubrication model when the distance gets too small;

(2) as the distance drops below some threshold value, the Stokes model, which is based on a continuous medium assumption, is no longer valid, and alternative models, which may make contacts possible, must be considered.

We propose a heuristic way to integrate the fact that contact may actually occur, which suppresses the memory effects as the distance drops below a certain value. We introduce a threshold value $\gamma_{0}$, and we add in the model the fact that $\gamma$ is not allowed to go below $\gamma_{0}$.

Let us now describe the way we plan to use this approach to model real situations. Considering a fluid with finite viscosity $\varepsilon>0$, and surfaces with roughness $e$, we consider that the model is valid for $q>2 e$, and that contact occurs as soon as $q=2 e$, which sets the value $\gamma_{0}$ at $\varepsilon \ln (2 e)$. Note that, from the numerical point of view, this new model is quite easy to implement by simply introducing a cut-off of $\gamma$ at $\gamma_{0}$.

\subsection{PDE version of this approach}

We would like to finish with some comments on what might be a continuous-eulerian version of what we did at the discrete-lagrangian level. In [8] we proposed a macroscopic model to represent the global behaviour of an array of spherical particles interacting through lubrication forces. Denoting by $\rho$ the macroscopic density of the solid phase ( $\rho=1$ when all spheres are in contact), the inertial version of the model we propose reads

$$
\begin{aligned}
\partial_{t} \rho+\partial_{x}(\rho u) & =0 \\
\partial_{t}(\rho u)+\partial_{x}\left(\rho u^{2}\right)-\partial_{x}\left(\frac{\varepsilon}{1-\rho} \partial_{x} u\right) & =f
\end{aligned}
$$

It is expected that a positive viscosity $\varepsilon$, as small as it may be, prevents the density $\rho$ to go above the saturation value 1 . As $\varepsilon$ goes to 0 , one can expect the system to behave like a pressureless gas with a unilateral constraint $\rho \leq 1$ (see [3,4]). Yet, as suggested by the present work, the limit behaviour is likely to exhibit a memory effect. It calls for the use of an extra variable, say $\gamma=\gamma(x, t)$, which can be expected to interact with the macroscopic velocity $u$ in the following way:

$$
\begin{aligned}
\partial_{t} \rho+\partial_{x}(\rho u) & =0 \\
\partial_{t}(\rho u)+\partial_{x}\left(\rho u^{2}\right)+\partial_{x} p & =f \\
\partial_{t} \gamma+\partial_{x}(\gamma u) & =-p \\
\gamma \leq 0, \quad \rho \leq 1, \gamma(1-\rho) & =0
\end{aligned}
$$

where $p$ is an unknown pressure-like field which may take negative values (which distinguishes this gluey model from the standard pressureless gas situation), and $\gamma$ is a field which keeps track of the constraint history experienced by a "fluid" particle, thus conveying its gluey character (note that $\rho$ is stuck to 1 as long as $\gamma$ is negative). Like for pressureless gases, such a system must be provided with a collision law. Without it, nothing prevents two saturated blocks $(\rho=1)$ to collide in an elastic shock, with a restitution coefficient which may take any nonnegative value. This collision law may be defined by prescribing that, at any time, the right velocity $u^{+}$is the $L^{2}$-projection of the left velocity $u^{-}$onto the closure in $L^{2}$ of

$$
C_{\rho \gamma}=\left\{v \in H^{1}, \partial_{x} v \geq 0 \text { a.e. on }[\rho=1, \gamma=0], \quad \partial_{x} v=0 \text { a.e. on }[\rho=1, \gamma<0]\right\} \text {. }
$$




\section{Concluding Remarks}

We presented a framework to model the near-contact behaviour of a rigid body and a wall, under the asumption that the interstitial gap remains filled with a viscous fluid. The approach we presented is quite straightforward from the mathematical standpoint. Yet, it raises some issues regarding modelling. We would like to conclude by addressing the most paradoxal among them. As indicated by numerical computations (see Remark 3.1), reasonably small values for $\varepsilon$ lead to values for the interstitial gap which are much smaller than atomistic distances, and a fortiori much smaller than usual roughness parameters. From a modelling standpoint, it clearly invalidates this model in the case of small viscosity, in accordance with common sense: a rigid ball projected onto a clean wall does not stick to it, although air may be considered in many situations as a viscous fluid. As the limit model is obtained by letting the viscosity go to 0 , one may wonder whether the limit model corresponds to any reality. Our belief (which has to be conforted by further analysis and experimental validation) is that, in a paradoxal way, this vanishing-viscosity model can be expected to have some validity in situations where the viscosity is large. Consider a physical situation, characterized by a given forcing term $f$, a threshold value $q_{\text {lim }}$ below which the lubrication model (1) does no longer hold (typically $q_{\text {lim }}$ is linked with the roughness of the surfaces), and a viscosity $\varepsilon$. If the viscosity is small in such a way that lubrication forces (as given by model (1)) become significant for values of $q$ smaller than $q_{\text {lim }}$, then our approach does not make sense. On the contrary, if $\varepsilon$ is large, one can expect $q$ to remain above $q_{\text {lim }}$, and the physical reality can be expected to exhibit the gluey behaviour we described.

\section{REFERENCES}

[1] P. Ballard, The Dynamics of Discrete Mechanical Systems with Perfect Unilateral Constraints, Arch. Rational Mech. Anal. 154, p.p. 199-274, 2000.

[2] I. Bazhlekov, F.N. van de Vosse, A.K.Chesters, Drainage and rupture of a newtonian film between two power-law liquid drops interacting under a constant force, J. Non-Newtonian Fluid Mech., 93, 181-201, 2000.

[3] F. Berthelin, Existence and weak stability for a pressureless model with unilateral constraint, Math. Models Methods Appl. Sci., 12, No 2, pp. 249-272, 2002.

[4] Y. Brenier, E. Grenier, Sticky particles and scalar conservation laws, SIAM J. Numer. Anal., 35, No 6, pp. 2317-2328 (electronic), 1998.

[5] Brenner, The slow motion of a sphere through a viscous fluid towards a plane surface, Chemical Engineering Science, 16, pp. 242-251, 1961.

[6] S. Dharmatti and M. A. Ramaswamy, Hybrid control systems and viscosity solutions, J SIAM J. Control Optim., 44, No 4, pp. 1259-1288 (electronic), 2005.

[7] S. Kim and S. J. Karrila, Microhydrodynamics: Principles and Selected Applications, Butterworth-Heinemann, Boston, 1991.

[8] A. Lefebvre, B. Maury, Micro-macro modelling of arrays of spheres interacting through lubrication forces, Prépublication du Laboratoire de Mathématiques de l'Université Paris-Sud 2005-46.

[9] B. Maury, A Many-Body Lubrication Model, C. R. Acad. Sci. Paris, t. 325, Série I, pp. 1053-1058, 1997.

[10] B. Maury, A time-stepping scheme for inelastic collisions, Numerische Mathematik, Volume 102, Number 4, pp. 649 - 679, 2006

[11] M. Schatzmann, A class of Nonlinear Differential Equations of Second Order in Time, Nonlinear Analysis, Theory, Methods \& Applications, 2, pp. 355-373, 1978.

[12] M. Schatzmann, Uniqueness and Continuous Dependence on Data for One Dimensional Impact Problems, Mathematical and Computational Modelling, 28, pp. 1-18, 1998. 